\section{Conclusion of Avery et al-Validation of Findings But Concern about Rationale}

To the Editor-Avery et al $^{1}$ recently utilized administrative data (diagnosis codes with present-on-admission [POA] indicators) to determine the number of patients with postdischarge-detected (post-DD) hospital-onset (HO) incident methicillin-resistant Staphylococcus aureus (MRSA) infection. After examining data from more than 1 million at-risk hospitalizations involving patients admitted to 27 hospitals in Orange County, California, during a 5-year period, the authors concluded that inclusion of patients with 30 -day postDD HO-MRSA tripled the median hospital incidence from 12.2 to 35.7 cases per 10,000 at-risk admissions and that inclusion of 1-year post-DD HO-MRSA increased the median hospital incidence more than 5-fold, to 66.5. Hospital ranking changed substantially when data on post-DD HO-MRSA cases were analyzed.

The Duke Infection Control Outreach Network (DICON) prospectively collects patient-specific surveillance data on patients from community hospitals identified as being colonized or infected with MRSA. Data from our 41 affiliated hospitals in 5 southeastern states allow us to determine rates of healthcare-acquired MRSA infection or colonization using the standard surveillance definitions utilized by most US hospitals. ${ }^{2}$ In addition, infection preventionists determine whether patients with MRSA infection were hospitalized within the preceding 1 year; whether they were directly admitted from home, another hospital, nursing home, hospice, or other extended care facility; and whether they were receiving dialysis prior to admission.

We used these prospectively collected surveillance data to validate and assess the accuracy of the estimates of the impact of including post-DD MRSA on the incidence of HO-MRSA made by Avery and colleagues.

During a 4-year period from January 1, 2008, through December 31, 2011, 670 cases of hospital-acquired MRSA infection were identified during a total of 6,206,222 patientdays at 26 DICON hospitals (rate, 10.8 cases per 100,000 patient-days). We identified an additional 4,397 cases of patients who were admitted with an incident MRSA infection within 1 year following a hospitalization. If we include these infections in our rate of hospital-acquired MRSA as performed by Avery and colleagues, the pooled rate of $\mathrm{HO}$ MRSA in our hospitals would increase 7.6-fold, to 81.6 cases per 100,000 patient-days.

Upon closer inspection of the article by Avery and colleagues and our data, however, we are concerned about the inclusion of some patients in this rate. Avery and colleagues note that $16 \%$ of patients with post-DD HO-MRSA were admitted from skilled nursing facilities with their incident MRSA infection, and $40 \%$ had been discharged to a skilled nursing facility after their previous admission. In our cohort, a total of 1385 (31\%) cases of MRSA within 1 year of hospital discharge were admitted from other facilities that could have just as likely been the source of MRSA exposure, including $922(21 \%)$ from a nursing home, $12(<0.1 \%)$ from a hospice, $162(4 \%)$ from another extended care facility, and $156(4 \%)$ from another acute care hospital. In addition, 399 (9\%) were receiving dialysis prior to their readmission. We disagree with the inclusion of these patient groups in rates of MRSA attributed to the acute care hospital, as these locations are also known risk factors for exposure to MRSA and other multidrug-resistant pathogens. ${ }^{3}$. When we exclude these patients from our rate of 1-year post-DD HO-MRSA, our rate remains 4.0-fold higher (2,665 cases; 42.9 cases per 100,000 patientdays).

Our data do not include information on whether individual patients had any contact with long-term care facilities during the preceding year but were discharged home prior to hospital readmission. Thus, our estimates are an underestimation of the true amount of exposure to skilled nursing facilities during the 1 year prior to incident MRSA infection.

In summary, surveillance data collected prospectively from patients in community hospitals affiliated with DICON in a more recent study period largely confirm the key conclusions of Avery and colleagues. Specifically, we agree that traditional surveillance methods greatly underestimate the true incidence of MRSA acquisition from the acute care hospital setting.

We do, however, feel it is important to point out the other important reservoirs of MRSA acquisition outside acute care facilities (eg, skilled nursing facilities and dialysis centers). In addition, Avery and colleagues reported that 5,302 (40\%) of incident MRSA infections were POA yes with no prior hospitalization, suggesting that a large part of MRSA cases in their study originated from a community source. Our data similarly confirm that $48 \%$ (5,186 cases) were community acquired by traditional surveillance definitions. Thus, whether any incident case can be reliably attributed to a specific healthcare facility and deemed not to be due to acquisition from a community reservoir could be an equally plausible concern, especially when hospitals do not routinely perform active surveillance.

Ranking hospitals by local incidence rates of MRSA that include postdischarge events is likely to be misleading and inaccurate. We would caution against using these data to rank hospitals on quality. Playing the blame game can be quite contentious, especially with a pathogen known to have strong endemicity outside acute care facilities. 


\section{ACKNOWLEDGMENTS}

Potential conflicts of interest. All authors report no conflicts of interest relevant to this article. All authors submitted the ICMJE Form for Disclosure of Potential Conflicts of Interest, and the conflicts that the editors consider relevant to this article are disclosed here.

Rebekah W. Moehring, MD; ${ }^{1}$ Daniel J. Sexton, MD; ${ }^{1}$ Deverick J. Anderson, MD, MPH

Affiliation: 1. Department of Medicine, Division of Infectious Diseases, Duke University Medical Center, Durham, North Carolina.

Address correspondence to Rebekah W. Moehring, MD, PO Box 102359, Department of Medicine, Division of Infectious Diseases, Duke University Medical Center, Durham, NC 27710 (rebekah.moehring@duke.edu).

Infect Control Hosp Epidemiol 2012;33(8):856-857

(C) 2012 by The Society for Healthcare Epidemiology of America. All rights reserved. 0899-823X/2012/3308-0016\$15.00. DOI: $10.1086 / 666633$

\section{REFERENCES}

1. Avery TR, Kleinman KP, Klompas M, Aschengrau A, Huang SS. Inclusion of 30-day postdischarge detection triples the incidence of hospital-onset methicillin-resistant Staphylococcus aureus. Infect Control Hosp Epidemiol 2012;33(2):114-121.

2. Cohen $\mathrm{AL}$, Calfee $\mathrm{D}$, Fridkin $\mathrm{SK}$, et al. Recommendations for metrics for multidrug-resistant organisms in healthcare settings: SHEA/HICPAC position paper. Infect Control Hosp Epidemiol 2008;29(10):901-913.

3. Klevens RM, Morrison MA, Nadle J, et al. Invasive methicillinresistant Staphylococcus aureus infections in the United States. JAMA 2007;298(15):1763-1771.

\section{Reply to Moehring et al}

To the Editor-The work of Moehring et $\mathrm{al}^{1}$ (hereafter, Moehring) is a welcome addition to the discussion of postdischarge-detected (post-DD) hospital-onset (HO) methicillin-resistant Staphylococcus aureus (MRSA). Their work highlights a valuable surveillance resource that allows for the tracking of patients with MRSA infections.

Although the analyses of Moehring tell a story similar to our work, it is important to note the differences in methodology. Moehring attributed post-DD HO-MRSA to hospitalizations that occurred up to 1 year prior to the detection of MRSA, whereas we limited the time frame for attribution to hospitalizations within 30 days prior to detection. In fact, $59 \%$ of hospitalizations in our study occurred within 2 weeks prior to the MRSA detection admission. This may help address Moehring's concern that our study may not represent healthcare-associated exposure. The brief interval between hospital discharge and evidence of MRSA suggests that the MRSA was likely healthcare associated and attributable to the recent hospital stay. Similarly, the brief interval makes it unlikely that community sources of MRSA were important sources of MRSA acquisition. We fully agree that calculating MRSA acquisition rates using a 1-year window for prior hospital exposure, such as is reflected in the analysis performed by Moehring, may well represent substantial community and healthcare exposures. An analysis of Moehring's data with a 30-day restriction would be interesting and would allow a more direct comparison between the 2 analyses.

In addition, Moehring mentions the fact that our population included substantial fractions of patients discharged to nursing homes and suggests that this may introduce additional important sources of MRSA acquisition. This is true. However, we note that our sensitivity analyses explicitly excluded individuals known to have had contact with a skilled nursing facility or acute inpatient stay between the MRSA acquisition admission (assigned by us) and the MRSA detection admission. This information was based on variables in the administrative data describing the discharge disposition and the source site prior to admission. We removed 1,237 (43\%) post-DD MRSA cases on the basis of the discharge location and $86(3 \%)$ on the basis of the next admission location. These results are described in our article. ${ }^{2}$

To address Moehring's concerns about patient contact with hemodialysis centers, we preformed an additional sensitivity analysis in which we reanalyzed the data excluding an additional $168(6 \%)$ post-DD cases occurring in people with renal disease on the basis of codes from the International Classification of Diseases, Ninth Revision. The results of this analysis showed that the inclusion of post-DD MRSA increased the median number of HO-MRSA cases per 10,000 at-risk admissions by a factor of 2.0 (12.2 to 24.4; $P=$ $.0003)$, compared with the 3.0 -fold increase when all patients were included ( 12.2 to $35.7 ; P<.0001)$. Thus, even when patients with other healthcare facility exposures were removed from the analysis, we found that MRSA acquisition was double what would otherwise be found within a hospital stay. This is supported by the assessment conducted by Moehring.

Another important difference between the work by Moehring and our study is that Moehring was able to identify and assess MRSA infections. For our own analysis, we were limited to MRSA carriage due to the known imperfections of administrative data in identifying MRSA infection. While there is evidence that MRSA carriage increases the risk of future infection, ${ }^{3}$ the identification of MRSA infection is more clinically meaningful, and therefore it is particularly important that Moehring found that significant amounts of MRSA infection come to light only after discharge.

In an era of public reporting of healthcare-associated infections, there is strong pressure to engage in a blame game, as mentioned by Moehring. However, the purpose of our study was not to focus on blaming hospitals but rather to galvanize policy makers and members of our field of infection prevention to join together to tackle the larger issue of a contagion that crosses hospital boundaries and is broadly shared across facilities. Our intent was to highlight the striking 